\title{
Scientific correspondence
}

\section{'Pontinization' of the medulla: two clinical case studies}

The anatomy of the pons has been extensively documented [1], and the developmental processes underlying its organization are a topic of intensive study (see [2] for a thorough review). Here, we document two examples of human autopsies where pontine nuclei and associated pontine projection fibres were found not only in the pons, but also in the rostral and mid-level medulla. In effect, the boundary between the ventral pons and ventral medulla is erased, creating 'pontinization' of the medulla. In contrast, dorsal pontine and medullary structures appear in normal register and do not overlap. Both cases show normal cranial nerve anatomy, and no other developmental abnormalities are found in the central nervous system. Neither patient was known to have any congenital neurological symptoms, including any signs of abnormal bulbar function. These findings suggest that although embryologic ponto-bulbar development normally proceeds in a stereotyped fashion, a remarkable degree of allowed variability exists which does not appear to have any detectable functional ramifications. This work has previously been presented in abstract form [3].

The first patient (patient A) was a 78-year-old man with a history of dilated cardiomyopathy, congestive heart failure and fibrotic lung disease. He was found unresponsive at his home with no detectable pulse. Emergency medical services restored a pulse, but after arrival at the emergency department, he developed pulseless electrical activity followed by asystole. An autopsy showed a hypertrophic and dilated cardiomyopathy and severe pulmonary fibrosis with superimposed bronchopneumonia, with signs of right-sided heart failure. In addition to the abnormality described in this paper, the brain autopsy showed signs of acute global ischaemia, as well as chronic infarctions in the left superior temporal gyrus and hippocampus, a small number of neurofibrillary tangles in the hippocampus, and a Lewy body in the locus ceruleus. However, it is important to recognize that patient A was not known to have any neurological symp- toms other than possible mild dementia; there were certainly no signs of abnormal bulbar function throughout his life.

The second patient (patient B) was originally reported as a case record in the New England Journal of Medicine [4]. This patient was an otherwise healthy 45-year-old woman who was recently diagnosed with Hodgkin's disease, nodular-sclerosis type. Within several weeks she began to develop a burning sensation as well as mild weakness in the distal extremities that soon progressed to total-body pain over the course of several days, with increased limb weakness as well as new bulbar symptoms (difficulty swallowing) and absent deep tendon reflexes. She died of cardiopulmonary arrest with associated aspiration, approximately 9 days after the onset of neurological symptoms. An autopsy revealed disappearance of nearly all the neurones in all the spinal sensory ganglia examined, as well as scattered lymphocytes within the interstitial tissues of the ganglia and their attached nerve fascicles, characteristic of an acute paraneoplastic sensory ganglionitis. Again, all of patient B's neurological symptoms were acute in onset; she showed no neurological abnormalities prior to the final few weeks of her life.

Both patients A and B showed a similar pattern of abnormal pontine anatomy at autopsy. Grossly, patient A's medulla is notable for being abnormally large, with a prominent, rounded basal surface. On axial cross section (Figure 1A), the medulla has clear inferior olivary nuclei that appear to be encased in firm white tissue. The firm white tissue composes the majority of the rounded, basal surface, and the pyramids are not clearly identified grossly. The pons and midbrain, by contrast, appear relatively normal. Histologically, the basal medulla is infiltrated by white matter tracts and nuclei identical to those normally seen in the pons (Figure 1B,C). These pontine tracts are interspersed around the pyramids, medial lemniscus and inferior olivary nuclei, all of which are normally orientated within the medulla. Note that the cranial nerve nuclei are in their proper locations (Figure 1B - see the hypoglossal nucleus, the dorsal motor nucleus of the vagus and the solitary nucleus and tract). The caudal pons 
A

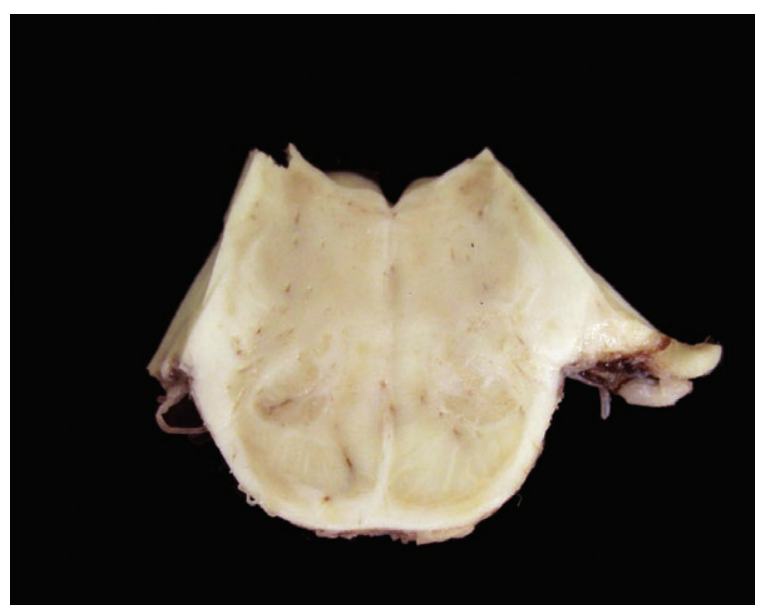

C

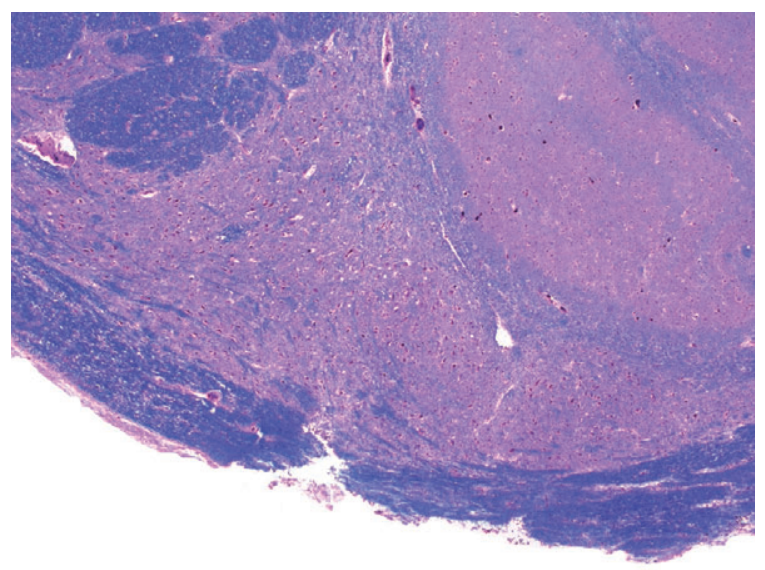

E

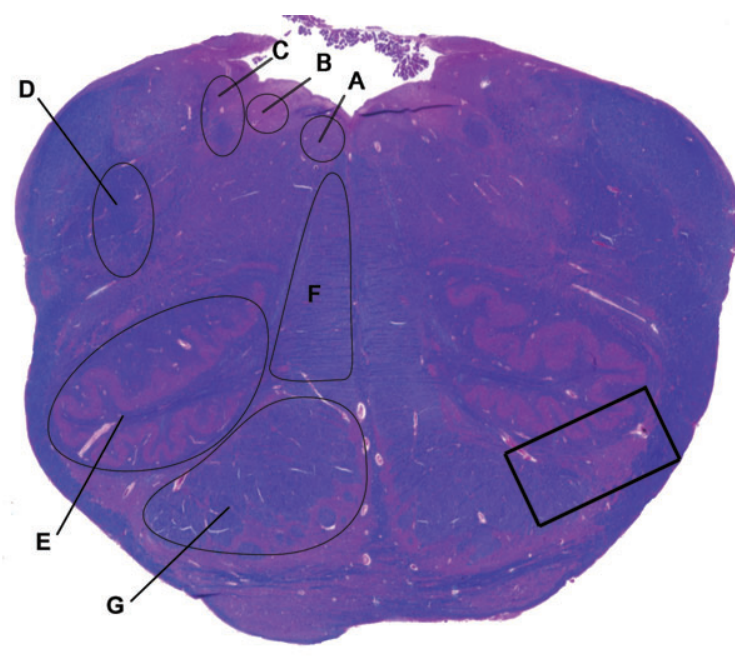

B

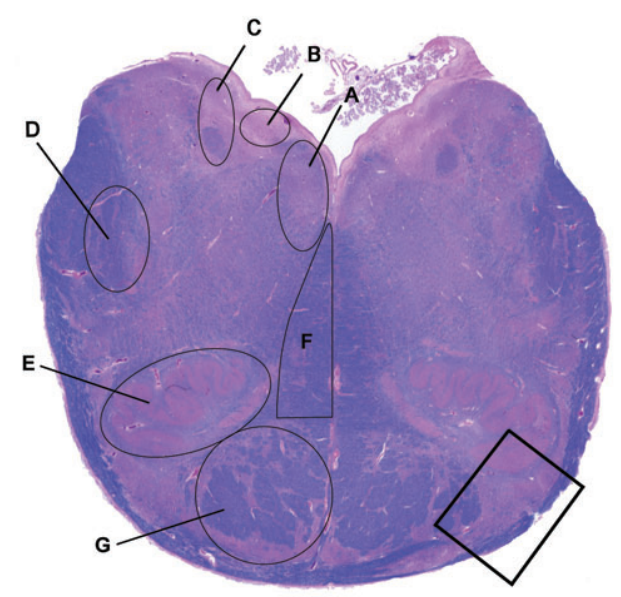

D
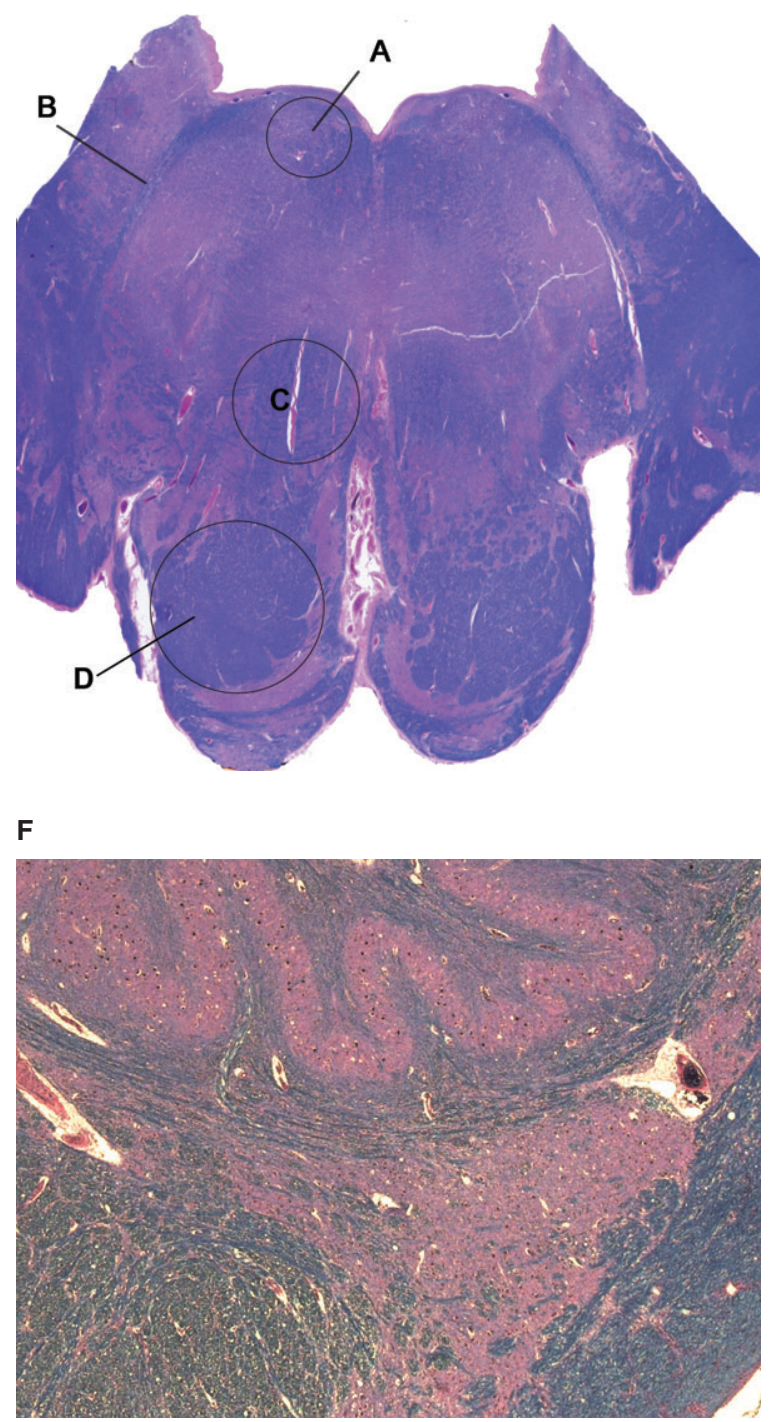

(C) 2011 The Authors Neuropathology and Applied Neurobiology (C) 2011 British Neuropathological Society, 37, 689-693 
(Figure 1D) has cortical-spinal tracts somewhat cohesive at the pontine base, appearing more like the pyramids in the medulla, with pontine nuclei and fibres surrounding these cortical-spinal tracts. Note that cranial nerve 7 loops normally around the nucleus of cranial nerve 6 dorsally. The rostral pons and midbrain, by contrast, are histologically unremarkable. We sectioned through the entire pons and medulla of patient $\mathrm{A}$, and despite the abnormal distribution of pontine fibres and pontine nuclei, the rest of the ponto-medullary anatomy appears completely normal, including normal positions of cranial nerve nuclei, normal exit points of the cranial nerves, and normal pyramid and medial lemniscus anatomy in the medulla. The abnormal pontine fibres and nuclei are present ventrally in every cross section of medulla, and are continuous with the neurones in the basal pons (see Supporting Information, Figures S1-S7 for a complete set of sections).

Patient B also has a medulla with pontine nuclei and pontine fibres intermixed between the pyramids, inferior olivary nuclei and medial lemniscus (Figure 1E,F). Here too, one can see the cranial nerve nuclei normally positioned along the dorsal surface as in patient $\mathrm{A}$ (that is, the hypoglossal nucleus, the dorsal motor nucleus of the vagus, and the solitary nucleus and tract). Neither of these two patients had any clinical history of congenital neurological abnormalities, including of any abnormal bulbar functioning. This suggests that 'pontinization' of the medulla may be a normal (albeit rare) anatomical variant of brainstem morphology that does not cause any clinical abnormalities.

The cause of this developmental variant remains unclear, and the development of the pons is a subject of ongoing study [2]. The embryological migration route of pontine neurones has been mapped out in rats $[5,6]$. Specifically, it has been shown in rats that tegmental pontine neurones derive from primary precerebellar neuroepithelium (which is thought to originate in the dorsomedial rhombencephalon), and are produced predominantly on days E15 and E16. These neurones migrate from a posterodorsal production site anteriorly by a subpial route on the lateral wall of the medulla and pons, and settle in a sequence from caudal (earlier generated neurones) to rostral (later generated neurones). The basal pontine neurones, by contrast, derive from the secondary precerebellar neuroepithelium, which forms on day E16 as an outgrowth of the primary precerebellar neuroepithelium, and remains mitotically active through day E19. The progenitors of basal pontine grey neurones follow the same subpial route as the tegmental progenitors, and form successive concentric layers in the basal pons, with the latest arriving cells forming the outermost 'shell' of neurones. Cortical-pontine fibres, in turn, derive from 'budding' off of axons that have already grown past the pons, a process caused by a diffusible factor (or factors) originating from the basal pons [7]. The two patients described here have basal pontine neurones and fibres located in the ventral medulla. Therefore, these patients might only need to have basal pontine progenitor neurones from secondary precerebellar neuroepithelium migrate into the medulla (perhaps caused by a derangement in factors that govern the formation of the pontine/medullary boundary) in order to have the resulting pontinization. The incoming cortical fibres would simply follow. Along these same lines, it is interesting to note that the inferior olivary nucleus is normally orientated in both patients. The progenitor

Figure 1. (For a complete set of figures, please see the online edition of this article, Figures S1-S7 under Supporting Information). Brainstem anatomy is shown for Patients A and B, documenting 'pontinization' of the medulla. (A) Horizontal section of medulla from Patient A. Note the rounded basal surface caused by an abnormal amount of firm white tissue. (B) Histological section of the medulla from Patient A reveals grey and white matter encasing the inferior olivary nuclei (E) and interdigitating within the pyramids (G). Note that the cranial nerve nuclei are all in normal register ( $\mathrm{A}$ - hypoglossal nucleus, B - dorsal motor nucleus of the vagus nerve, $\mathrm{C}-$ solitary tract and nucleus) as is the spinal trigeminal tract (D) and the medial lemniscus (F). This section is taken at $+6 \mathrm{~mm}$ from the obex according to the brainstem atlas of Paxinos and Huang [11]. Luxol-fast blue, haematoxylin and eosin stain (original magnification $-1 \times$ ). (C) Higher power view of the boxed region in panel B $(2.5 \times$ objective; $25 \times$ magnification). (D) Section of caudal pons from Patient A shows that the seventh cranial nerve (B) arches around the nucleus of the sixth cranial nerve (A), in the normal manner. The medial lemniscus (C) and corticospinal tracts (D) are also shown. This section is taken at $+16 \mathrm{~mm}$ from the obex according to the brainstem atlas of Paxinos and Huang [11]. Luxol-fast blue, haematoxylin and eosin stain (original magnification - 1×). (E) Section of medulla from Patient B. Note the grey and white matter tissue encasing the inferior olivary nuclei (E) and interdigitating within the pyramids (G), similarly to Patient A (compare with panel B). Note that the cranial nerve nuclei are all in normal register (A - hypoglossal nucleus, B - dorsal motor nucleus of the vagus nerve, $\mathrm{C}$ - solitary tract and nucleus) as is the spinal trigeminal tract (D) and the medial lemniscus (F). This section is taken at $+5 \mathrm{~mm}$ from the obex according to the brainstem atlas of Paxinos and Huang [11]. Luxol-fast blue, haematoxylin and eosin stain (original magnification $-1 \times)$. (F) Higher power view of the boxed region in panel E $(2.5 \times$ objective; $25 \times$ magnification $)$.

(C) 2011 The Authors

Neuropathology and Applied Neurobiology (C) 2011 British Neuropathological Society, 37, 689-693 
neurones of the inferior olivary nucleus originate several days earlier but at the same site as basal pontine progenitor neurones [5], and like basal pontine neurones, the inferior olivary neurones also connect to the cerebellum. The fact that the inferior olivary nuclei appear normal in these patients is consistent with the idea that these patients only have a disruption in the formation in the pontine/medullary boundary, and not in the formation of neurones from the dorsomedial rhombencephalon.

Several pathological conditions are known to disrupt pontine neurone migration $[8,9]$, but these conditions result in pronounced neurological derangement, in contrast to our two cases. We have also considered whether some of the 'pontine' neurones we see in our two patients may be enlarged or accessory arcuate nuclei. The arcuate nucleus derives from the same rhombic lip region as pontine neurones [10], and we do not have a clear way of distinguishing between the two possibilities of ectopic pontine neurones vs. ectopic arcuate neurones. However, given the large number of neurones we see in the ventral medulla and the continuity of these neuronal clusters with the ventral pons, we are inclined to assume the former.

\section{Acknowledgements}

We would like to thank Dr Jean Paul Vonsattel for helping us with connecting the two cases presented here, as well as for helping us prepare the photographs of the histological sections. We would also like to thank Dr Hannah Kinney and Dr Trygve Leergaard for helpful comments during the preparation of the manuscript.

\section{Conflict of interest}

The authors declare no conflicts of interest.

$$
\begin{array}{r}
\text { A. F. Teich* } \\
\text { E. T. Hedley-Whyte† } \\
\text { J. E. Goldman* } \\
\text { *Department of Pathology and Cell Biology, Columbia } \\
\text { University Medical Center and the New York Presbyterian } \\
\text { Hospital, New York, NY, and †Department of Pathology - } \\
\text { Neuropathology, Harvard Medical School - Massachusetts } \\
\text { General Hospital, Boston, MA, USA }
\end{array}
$$

\section{References}

1 Carpenter M, Sutin J. Human Neuroanatomy, 8th edn. Baltimore, MD: Williams and Wilkins, 1981
2 Leergaard TB. Clustered and laminar topographic patterns in rat cerebro-pontine pathways. Anat Embryol 2003; 206: 149-62

3 Teich AF, Hedley-Whyte ET, Goldman JE. 'Pontinization' of the medulla: two clinical case studies. In AANP 2009 Annual Meeting: JNEN. 2009: 577

4 Scully RE, Mark EJ, McNeely WF, McNeely BU. Case records of the Massachusetts General Hospital. Weekly clinicopathological exercises. Case 8-1990. A 45-year-old woman with Hodgkin's disease and a neurologic disorder. N Engl J Med 1990; 322: 531-43

5 Altman J, Bayer SA. Prenatal development of the cerebellar system in the rat. II. Cytogenesis and histogenesis of the inferior olive, pontine gray, and the precerebellar reticular nuclei. J Comp Neurol 1978; 179: 49-75

6 Altman J, Bayer SA. Development of the precerebellar nuclei in the rat: IV. The anterior precerebellar extramural migratory stream and the nucleus reticularis tegmenti pontis and the basal pontine gray. J Comp Neurol 1987; 257: 529-52

7 Heffner CD, Lumsden AG, O'Leary DD. Target control of collateral extension and directional axon growth in the mammalian brain. Science 1990; 247: 217-20

8 Yamamoto M, Zhang J, Smith D, Hayakawa Y, McCaffery P. A critical period for retinoic acid teratogenesis and loss of neurophilic migration of pontine nuclei neurons. Mech Dev 2003; 120: 701-9

9 Saito Y, Kobayashi M, Itoh M, Saito K, Mizuguchi M, Sasaki H, Arima K, Yamamoto T, Takashima S, Sasaki M, Hayashi K, Osawa M. Aberrant neuronal migration in the brainstem of Fukuyama-type congenital muscular dystrophy. J Neuropathol Exp Neurol 2003; 62: 497-508

10 Essick CR. The development of the nuclei pontis and the nucleus arcuatus in man. Am J Anat 1912; 13: 25-54

11 Paxinos G, Xu-Feng H. Atlas of the Human Brainstem. San Diego: Academic Press, 1995

\section{Supporting information}

Additional Supporting Information may be found in the online version of this article:

Figure S1. Horizontal section of medulla from Patient A. Note the rounded basal surface caused by an abnormal amount of firm white tissue.

Figure S2. Horizontal section of pons from Patient A. In contrast to the medulla, the pons is grossly unremarkable. Figure S3. Histological sections of the medulla from Patient A. (A) Low power view reveals gray and white matter encasing the inferior olivary nuclei (E) and interdigitating within the pyramids $(G)$. Note that the cranial nerve nuclei are all in normal register (A - hypoglossal nucleus, $\mathrm{B}-$ dorsal motor nucleus of the vagus nerve, $\mathrm{C}$ 
- solitary tract and nucleus) as is the spinal trigeminal tract (D) and the medial lemniscus (F). This section is taken at $+6 \mathrm{~mm}$ from the obex according to the brainstem atlas of Paxinos and Huang [11]. Luxol-fast blue, haematoxylin and eosin stain (original magnification $1 \times)$. (B) Higher power view of the boxed region in panel 3 A $(2.5 \times$ objective; $25 \times$ magnification).

Figure S4. Additional histological sections of medulla from Patient A. These sections are rostral to the sections in Figure S3. (A) Low power view shows that abnormal pontine nuclei and fibers are still seen encasing the inferior olivary nuclei and pyramids, despite otherwise normal brainstem architecture (A - prepositus nucleus, $\mathrm{B}$ - the spinal trigeminal tract, $\mathrm{C}$ - the medial lemniscus, D - inferior olivary nucleus, E - pyramidal tract). This section is taken at $+11 \mathrm{~mm}$ from the obex according to the brainstem atlas of Paxinos and Huang [11]. Luxolfast blue, haematoxylin and eosin stain (original magnification $-1 \times$ ). (B) Higher power view of the boxed region in panel $4 \mathrm{~A}$ ( $2.5 \times$ objective; $25 \times$ magnification).

Figure S5. Sections of caudal pons from Patient A. (A) Low power view shows that the seventh cranial nerve (B) arches around the nucleus of the sixth cranial nerve (A), in the normal manner. The medial lemniscus (C) and corticospinal tracts (D) are also shown. This section is taken at $+16 \mathrm{~mm}$ from the obex according to the brainstem atlas of Paxinos and Huang [11]. Luxol-fast blue, haematoxylin and eosin stain (original magnification $-1 \times$ ). (B) Higher power view of the boxed region in panel $5 \mathrm{~A}$ (2.5× objective; $25 \times$ magnification).

Figure S6. Histological sections of pons from Patient A. (A) Low power view shows that the basal nuclei and fibers are normally distributed, anatomically continuous with the basal nuclei and fibres seen in the preceding sections
(Figures S3-S5). The pons is histologically unremarkable, with normal architecture (A - medial longitudinal fasciculus, B - locus coeruleus, C - superior cerebellar peduncle, $\mathrm{D}$ - central tegmental tract, $\mathrm{E}$ - medial lemniscus). This section is taken at $+25 \mathrm{~mm}$ from the obex according to the brainstem atlas of Paxinos and Huang [11]. Luxol-fast blue, haematoxylin and eosin stain (original magnification $-1 \times)$. (B) Higher power view of the boxed region in panel $6 \mathrm{~A}(2.5 \times$ objective; $25 \times$ magnification).

Figure S7. Sections of medulla from Patient B. (A) Low power view. Note the grey and white matter tissue encasing the inferior olivary nuclei (E) and interdigitating within the pyramids $(G)$, similarly to Patient A (compare with Figure S3A). Note that the cranial nerve nuclei are all in normal register (A - hypoglossal nucleus, B - dorsal motor nucleus of the vagus nerve, $\mathrm{C}$ - solitary tract and nucleus) as is the spinal trigeminal tract (D) and the medial lemniscus (F). This section is taken at $+5 \mathrm{~mm}$ from the obex according to the brainstem atlas of Paxinos and Huang [11]. Luxol-fast blue, haematoxylin and eosin stain (original magnification $-1 \times$ ). (B) Higher power view of the boxed region in panel $7 \mathrm{~A}(2.5 \times$ objective; $25 \times$ magnification).

Please note: Wiley-Blackwell are not responsible for the content or functionality of any supporting materials supplied by the authors. Any queries (other than missing material) should be directed to the corresponding author for the article.

Received 21 July 2010

Accepted after revision 2 March 2011

Published online Article Accepted on 31 March 2011 